\title{
Osteotomia Le Fort I no tratamento da nasoangiofibroma juvenil: uma revisão de
}

\section{literatura}

\author{
Le Fort I osteotomy in the treatment of juvenile nasoangiofibroma: a literatura review
}

Osteotomía Le Fort I en el tratamiento del nasoangiofibroma juvenil: revisión de la literatura

Camila Holanda Cavalcante Matos

ORCID: https://orcid.org/0000-0001-8283-0590 Centro Universitário Cesmac, Brasil

E-mail: camilaacavalcantem@outlook.com

Lorena Gabrielle Alves Teixeira

ORCID: https://orcid.org/0000-0002-7561-3528 Centro Universitário Cesmac, Brasil E-mail: lorenateixeirag@hotmail.com

Flávia de Paiva Teixeira Barros ORCID: https://orcid.org/0000-0001-6545-9021 Centro Universitário Cesmac, Brasil E-mail: flaviapaivatbarros@gmail.com

Gabrielly Maria Argolo Acioly ORCID: https://orcid.org/0000-0003-4248-7892 Centro Universitário Cesmac, Brasil E-mail: gaabiargolo@hotmail.com

Amanda Kelly de Carvalho Barros ORCID: https://orcid.org/0000-0001-7003-9830 Centro Universitário Cesmac, Brasil E-mail: amandakcb16@gmail.com

Micael Luiz Sabino dos Santos ORCID: https://orcid.org/0000-0003-0701-8062 Centro Universitário Cesmac, Brasil E-mail: micaelsabino@hotmail.com

Luiz Henrique Carvalho Batista ORCID: https://orcid.org/0000-0003-3249-145X Centro Universitário Cesmac, Brasil E-mail: luiz.batista@cesmac.edu.br

Lucas Fortes Cavalcanti de Macedo ORCID: https://orcid.org/0000-0002-8380-6442 Centro Universitário Cesmac, Brasil E-mail: lucas.macedo@cesmac.edu.br

\begin{abstract}
Resumo
O nasoangiofibroma juvenil (NAJ) é um tumor nasofaríngeo raro, benigno, vascularizado e acomete principalmente jovens do gênero masculino. Há uma predileção em indivíduos entre 14 e 25 anos de idade, correspondendo a $0,05 \%$ a $0,5 \%$ entre os tumores da cabeça e pescoço. O tratamento de escolha mais indicado é o procedimento cirúrgico, dentre os quais, a osteotomia Le Fort I é uma das alternativas, possibilitando manuseio da região da neoplasia, bem como do pedículo vascular. Sendo assim, o objetivo desse estudo será investigar o tratamento da osteotomia Le Fort I no nasoangiofibroma juvenil. Trata-se de uma revisão de literatura narrativa, de caráter descritivo e abordagem quantitativa, realizada mediante levantamento nas bases de dados eletrônicas como SciELO, BVS, PubMed, Scholar Google e Elsevier, utilizando os DeCS: "osteotomia", "terapêutica", "angiofibroma", os quais foram combinados por meio dos operadores booleanos $A N D$ e $O R$. Como critérios de elegibilidade, foram adotados artigos em português, inglês ou espanhol e estudos que possuíam integração com nasoangiofibroma juvenil e osteotomia Le Fort I. O tratamento proposto permite que os acessos transmaxilares possam ser realizados com confiança, pois apresenta baixas complicações. Somado a isso, uma das vantagens desse procedimento é a redução do tempo na cirurgia, quando comparado a cirurgias craniofaciais e ressecções endoscópias. A osteotomia Le Fort I mostrou-se eficaz e segura no tratamento do nasoangiofibroma juvenil, pois proporciona amplo acesso cirúrgico, com visão direta e palpação às estruturas, sendo uma alternativa para o tratamento da patologia.
\end{abstract}

Palavras-chave: Osteotomia; Terapêutica; Angiofibroma. 


\begin{abstract}
Juvenile nasoangiofibroma (NAJ) is a rare, benign, vascularized nasopharyngeal tumor that affects mainly young males. There is a predilection in individuals between 14 and 25 years of age, corresponding to $0.05 \%$ to $0.5 \%$ among head and neck tumors. The most indicated treatment of choice is the surgical procedure, among which the Le Fort I osteotomy is one of the alternatives, enabling the handling of the neoplasm region, as well as the vascular pedicle. Therefore, the aim of this study will be to investigate the treatment of Le Fort I osteotomy in juvenile nasoangiofibroma. This is a narrative literature review, with a descriptive character and a quantitative approach, carried out by surveying electronic databases such as SciELO, BVS, PubMed, Scholar Google and Elsevier, using the DeCS: "osteotomy", "therapeutics", " angiofibroma", which were combined using the Boolean operators AND and OR. As eligibility criteria, articles in Portuguese, English or Spanish and studies that had integration with juvenile nasoangiofibroma and Le Fort I osteotomy were adopted. The proposed treatment allows transmaxillary access to be performed with confidence, as it has low complications. Added to this, one of the advantages of this procedure is the reduction in surgery time, when compared to craniofacial surgeries and endoscopic resections. Le Fort I osteotomy proved to be effective and safe in the treatment of juvenile nasoangiofibroma, as it provides wide surgical access, with direct vision and palpation to the structures, being an alternative for the treatment of the pathology.
\end{abstract}

Keywords: Osteotomy; Therapeutics; Angiofibroma.

\title{
Resumen
}

El nasoangiofibroma juvenil (NAJ) es un tumor nasofaríngeo vascularizado benigno y poco común que afecta principalmente a hombres jóvenes. Existe una predilección en individuos entre 14 y 25 años, que corresponde al $0,05 \%$ al $0,5 \%$ entre los tumores de cabeza y cuello. El tratamiento de elección más indicado es el procedimiento quirúrgico, entre los que la osteotomía Le Fort I es una de las alternativas, que permite el manejo de la región neoplásica, así como del pedículo vascular. Por tanto, el objetivo de este estudio será investigar el tratamiento de la osteotomía Le Fort I en nasoangiofibroma juvenil. Se trata de una revisión de literatura narrativa, de carácter descriptivo y con abordaje cuantitativo, realizada por relevamiento de bases de datos electrónicas como SciELO, BVS, PubMed, Scholar Google y Elsevier, utilizando DeCS: "osteotomía", "terapéutica", "angiofibroma", que fueron combinados por los operadores booleanos AND y OR. Como criterios de elegibilidad se adoptaron artículos en portugués, inglés o español y estudios que tuvieran integración con nasoangiofibroma juvenil y osteotomía Le Fort I. El tratamiento propuesto permite realizar el acceso transmaxilar de forma segura, ya que presenta bajas complicaciones. Sumado a esto, una de las ventajas de este procedimiento es la reducción del tiempo quirúrgico, en comparación con las cirugías craneofaciales y resecciones endoscópicas. La osteotomía Le Fort I demostró ser eficaz y segura en el tratamiento del nasoangiofibroma juvenil, ya que proporciona un amplio acceso quirúrgico, con visión directa y palpación de las estructuras, siendo una alternativa para el tratamiento de la lesión. hología.

Palabras clave: Osteotomía; Terapéutica; Angiofibroma.

\section{Introdução}

O nasoangiofibroma juvenil (NAJ) é um tumor nasofaríngeo raro, histologicamente benigno, altamente vascularizado e acomete principalmente adolescentes do sexo masculino (Correa et al., 2011; Quiquia, 2017). Este tipo de tumor tem início no forame esfenopalatino, que está localizado na nasofaringe, onde está a artéria de mesmo nome; seu crescimento é assintomático e varia conforme seu tamanho (Acevedo et al., 2018; Peixes, 2019). Durante o processo de crescimento, o NAJ invade as cavidades paranasais, orofaringe, fossa intratemporal e o pterigomaxilar por contiguidade, órbita pelas fissuras orbitárias e base média do crânio, comprometendo o seio cavernoso, quiasma óptico e a hipófise, incluindo o craniano anterior fossa, sendo nesses casos intracraniana, mas extrameningeal (Iglesias et al., 2017; Acevedo et al., 2018).

Conforme estudo realizado por Martins et al. (2013) e Viana Júnior (2018), há uma incidência em indivíduos entre 14 e 25 anos de idade, correspondendo a $0,05 \%$ a $0,5 \%$ entre os tumores da cabeça e pescoço. Apesar da sua etiologia ser desconhecida, considera-se que há influência hormonal androgênica, com ênfase nos mecanismos moleculares envolvidos, congênita, vascular e genética (Hintz et al., 2018). As características clínicas mais frequentes podem ser a obstrução nasal e epistaxe, sendo as menos comuns o corrimento nasal, dor, sinusite e problemas auditivos. Seu diagnóstico consiste nos achados clínicos associados aos exames de imagem, tendo em vista que a biópsia incisional é dificultada devido as características vasculares da doença (Catunda et al., 2011; Viana Júnior et al., 2018; Hechavarría et al., 2020). 
Sob essa perspectiva, Viana Júnior et al. (2018) afirmam que a tomografia computadorizada, ressonância nuclear magnética e estudos angiográficos são de suma importância para este diagnóstico e na sua classificação, assim, auxiliando no método terapêutico.

Em relação às classificações existentes, Salcedo e Buitrago (2011) enfatizam duas mais frequentes: Fisch e Andrew. Estas, fundamentam-se, sobretudo, na extensão da doença e envolvimento das estruturas circunvizinhas, que estão comprometidas com a patologia. A classificação de Fisch em 1983 considera: Estágio I - Tumor limitado à nasofaringe e cavidade nasal sem destruição óssea; Estágigo II - Tumor invade a fossa pterigomandibular, seios paranasais com destruição óssea; Estágio III - Tumor invade a fossa infratemporal, órbita e região parasselar permanecendo lateral ao seio cavenoso; Estágio IV - Um tumor com invasão maciça do seio cavernoso, região do quiasmo óptico ou fossa pituitária.

A classificação de Andrew em 1989 por sua vez, considera: Estágio I - Limitado à nasofaringe e cavidade nasal sem destruição óssea ou limitado ao forame esfenopalatino; Estágio II - Invade a fossa pterigopalatina ou um dos seios maxilar, etmóide ou esfenoidal com destruição óssea; Estágio IIIa - Invade a fossa infratemporal ou órbita sem envolvimento intracraniano; Estágio IIIb-Invade a fossa infratemporal ou órbita com invasão intracraniana extradural e parasselar; Estágio IVa - Tumor intradural intracraniano sem infiltração do seio cavernoso, fossa hipofisária ou quiasma óptico; Estágio IVb- Tumor intradural intracraniano com infiltração do seio cavernoso, fossa pituitária ou quiasma óptico (Salcedo \& Buitrago, 2011).

A excisão cirúrgica em associação com a embolização prévia do tumor é vista como tratamento de eleição para esta entidade, no entanto, há inúmeros procedimentos cirúrgicos, como a via transplantal, ritonomia lateral, degloving médio-facial ou osteotomia Le Fort I. Dentre os tratamentos, a osteotomia Le Fort I é o mais indicado, tendo em vista que há uma exposição tumoral por meio do acesso direto e amplo aos seios maxilares e etmoidais, possibilitando manuseio da região do tumor, bem como do pedículo vascular (Chavolla-Maganã et al., 2019; Torres et al., 2012; Viana Júnior et al., 2018).

Apesar de ser um procedimento seguro, o tratamento não está isento de complicações e sequelas (Godoy et al., 2013; Acevedo et al., 2018). Algumas complicações como lesão aos nervos cranianos, amaurose, sangramento abundante, fraturas na base do crânio foram mencionadas e, algumas sequelas incluem infecções, defeitos periodontais, alterações pulpares e perda parcial ou completa da maxila (Quintão et al., 2019).

Diante do exposto, o objetivo deste estudo será investigar o tratamento da osteotomia Le Fort I no nasoangiofibroma juvenil, bem como analisar as vantagens e desvantagens dessa técnica cirúrgica.

\section{Metodologia}

O referido estudo seguiu os princípios de uma revisão de literatura narrativa, de caráter descritivo, de abordagem quantitativa. No que se refere aos procedimentos técnicos, este, caracterizou-se como uma pesquisa bibliográfica, desenvolvida com base em material já elaborado, constituído principalmente de artigos científicos e livros. A análise e síntese dos dados se seguiram através das seguintes etapas propostas por Santos e Parra Filho (2012): leitura, reflexão, análise, diferenciação, comparação e apontamentos. A análise textual teve por finalidade básica proporcionar uma revisão atualizada acerca do tratamento do nasoangiofibrona juvenil por meio da osteotomia Le Fort I.

Para a realização do estudo foram consultadas as bases de dados eletrônicas: Scientific Eletronic Library Online (SciELO), Biblioteca Virtual em Saúde (BVS), National Library of Medicine (PubMed), Scholar Google, Elsevier. Para se realizar tal busca, foram utilizados os seguintes Descritores em Ciência da Saúde (DeCS): "osteotomia", "terapêutica", "angiofibroma", nos idiomas inglês, espanhol e português, os quais foram combinados através dos operadores booleanos $A N D$ e $O R$ a fim de refinar a pesquisa e proporcionar maior qualidade à mesma. 
Foram selecionadas 24 referências para a confecção do estudo. Para a coleta de dados, foram considerados artigos científicos com limite temporal entre os anos de 2008 e 2021. Como critérios de elegibilidade, foram adotados artigos publicados em português, inglês ou espanhol e estudos que possuíam integração com nasoangiofibroma juvenil e osteotomia Le Fort I. Dessa forma, excluindo todos aqueles que não condiziam com o tema que foi proposto ou que fossem redundantes.

\section{Resultados e Discussão}

A osteotomia Le Fort I é um procedimento cirúrgico, descrito como uma fratura horizontal de abertura piriforme ao processo pterigoide da maxila bilateralmente (Catunda et al., 2011). Essa técnica permite acessar a cavidade nasal, seios paranasais, nasofaringe e orofaringe, região geniana, fossa pterigomaxilar, fossa zigomáticos, base do crânio e espaços anatômicos, à medida que são ocupados pelo tumor. No entanto, apresenta limitação na região do seio cavernoso, quiasma e hipófise, cujo são melhores abordados por meio de uma craniotomia (Acevedo et al., 2018).

Para o tratamento do nasoangiofibroma juvenil, os acessos transmaxilares podem ser empregados com segurança, pois apresenta poucas complicações e proporciona acesso direto à base do crânio (Catunda et al., 2011).

No entanto, alguns autores (quadro 1) relatam que embora seja raro, há riscos que devem ser avaliados.

Quadro 1 - complicações decorrentes da Osteotomia de Le Fort I

\begin{tabular}{|c|c|}
\hline Autor/Ano/Título & Complicações \\
\hline $\begin{array}{l}\text { Osteotomia le fort i: aspectos de interesse no tratamento de } \\
\text { nasoangiofibroma juvenil } \\
\text { Catunda et al. (2011) }\end{array}$ & $\begin{array}{l}\text { Pode-se destacar hemorragia pós-operatória, enfisema } \\
\text { subcutâneo, necrose avascular maxilar, comprometimento } \\
\text { oftalmológico, fístula do seio cavernoso e fraturas } \\
\text { indesejadas. }\end{array}$ \\
\hline $\begin{array}{l}\text { Complicações peri e pós operatórias em osteotomias Le Fort } \\
\text { I }\end{array}$ & $\begin{array}{l}\text { As complicações peri operatórias variam de } 6,4 \% \text { a } 7 \% \text {, } \\
\text { enquanto as complicações pós operatórias variam de } 1,8 \% \text { a } \\
27 \% \text { em osteotomias segmentares e } 4 \% \text { e } 11,7 \% \text { em } \\
\text { osteotomias convencionais. Entretanto, essas complicações } \\
\text { são leves e transitórias. Entre as complexidades no peri } \\
\text { operatório, pode-se destacar: comunicação oronasal em } 6 \% \\
\text { dos pacientes, rompimento da mucosa em } 8,7 \% \text { das } \\
\text { osteotomias segmentares e } 1,3 \% \text { em osteotomias de peça } \\
\text { única, } 2 \% \text { de hemorragia, 1\% em desdobramento } \\
\text { desfavorável e } 1 \% \text { de complicações dentais. }\end{array}$ \\
\hline $\begin{array}{l}\text { Osteotomia Le Fort I para angiofibroma nasofaríngeo juvenil } \\
\text { extenso: um estudo retrospectivo } \\
\text { (Lin et al. 2008) }\end{array}$ & $\begin{array}{l}\text { As possíveis complicações podem ser amaurose, enfisema } \\
\text { subcutâneo, desoclusão, enoftalmia, perda de dentes, lesão do } \\
\text { nervo abducente e necrose asséptica da maxila. }\end{array}$ \\
\hline $\begin{array}{l}\text { Ressecção de nasoangiofibroma juvenil por osteotomia Le } \\
\text { Fort I: Experiência com } 40 \text { casos }\end{array}$ & $\begin{array}{l}\text { As complicações operatórias incidiram em } 15 \% \text { dos casos, } \\
\text { entre elas, estão: infecção de ferida operatória }(2,5 \%) \text {, } \\
\text { extrusão tardia do material de síntese }(2,5 \%) \text {, má oclusão } \\
(5 \%) \text {, sangramento intra-operatório abundante }(5 \%) \text {, um } \\
\text { acidente vascular isquêmico. }\end{array}$ \\
\hline Lesão do nervo cranianos após osteotomia Le Fort I & $\begin{array}{l}\text { Várias desordens foram explanadas após a cirurgia. } \\
\text { Complicações sérias estão relacionadas a danos aos nervos. } \\
\text { Lesões dos nervos cranianos, como lesões dos nervos ópticos } \\
\text { (NC II), oculomotores (NCIII) e abducentes (NC VI), podem } \\
\text { ocorrer após a osteotomia Le Fort I. }\end{array}$ \\
\hline
\end{tabular}

Fonte: Autoria própria (dados da pesquisa).

No estudo de Kotaniemi et al. (2021), ele sustenta que essa técnica pode acometer a saúde dos seios paranasais, em consequência da delimitação da área dos seios, variações morfológicas e encadeamentos inflamatórios. Podem ser encontrados por meio de um exame radiográfico ou endoscópico das áreas etmoidais no pré e pós-operatório, podendo desencadear informações em decorrência de algum processo inflamatório existente. Visto que, devido ao comprometimento das áreas 
relacionada aos seios da face, é comum uma incidência de sinusite pós-operatória, sendo visto em portadores com sintoma subjetivo.

Em contrapartida, Hernández-Alfaro e Guijarro-Martínez (2013) certificam que é um procedimento seguro, confiável, previsível e minimamente invasivo. Ainda assim, garantem que esta abordagem é executável nas condições do paciente e precisão técnica, pois os pacientes submetidos ao procedimento não apresentaram complicações significativas ou fraturas iatrogênicas expostas. Analogamente a isso, Catunda et al. (2015) confirma que é uma via segura, aprimorando o processo das neoplasias da nasofaringe e da base do crânio, quando recomendada. Os riscos são raros e é executado com aptidão, com campo cirúrgico amplo, alcance para tumores volumosos, diminuindo as chances de recidiva.

Consoante a isso, Mello-Filho et al. (2015) afirma que uma das vantagens desse procedimento é a redução do tempo de cirurgia, quando comparado a cirurgias craniofaciais e ressecções endoscópicas. Atrelado a isso, dentre os participantes com NAJ, ocorreu complicações em 15\% dos casos, sendo o sangramento intra-operatório o mais comum, e recidivas em 5\% dos casos, cujo podem ser evitadas por meio do acesso transfacial em conjunto com a via endoscópica.

Viana Júnior et al. (2018) enfatiza a importância da embolização preventiva dos vasos mantenedores para evitar sangramentos transoperatórios, sendo realizada 24 horas antes da intervenção cirúrgica. $\mathrm{O}$ estudo enfatiza também os benefícios da Osteotomia Le Fort I, sendo: pequena excisão do tecido ósseo; redução de lesões neurovasculares; ajuda na estética, tendo em vista que o acesso é intrabucal.

A conduta a ser seguida por Correa et al. (2011) em casos de recidiva é a radioterapia, pois possui resultados positivos quando a lesão possui extensão intracraniana ou recorrentes.

Como alternativa à osteotomia Le Fort I, a endoscopia tem se tornado uma opção recorrente entre os otorrinolaringologistas, principalmente no público jovem, uma vez que a osteotomia pode causar danos no desenvolvimento da face. Contudo, é recomendável apenas em estágios iniciais do NAJ e é imprescindível especialistas na área, que nem sempre está disponível aos pacientes que usufruem do Sistema Único de Saúde (Viana Júnior et al., 2018; González et al., 2020).

\section{Considerações Finais}

A osteotomia Le Fort I mostrou-se eficaz e segura no tratamento do nasoangiofibroma juvenil, pois proporciona amplo acesso cirúrgico, com visão direta e palpação às estruturas, sendo uma alternativa pra o tratamento da patologia. Durante o procedimento cirúrgico e até mesmo no pós-operatório pode haver complicações e recidivas, embora não seja comum. Devese enfatizar, portanto, a importância do aprofundamento técnico do cirurgião e saber como resolvê-los.

Por fim, recomenda-se para trabalhos futuros, a necessidade da realização de novos estudos que visem a melhor compreensão da osteotomia le fort I no tratamento do NAJ. Sendo assim, um diagnóstico direto durante o exame físico resulta, na maioria dos casos, em um método mais conservador e de sucesso durante o tratamento.

\section{Referências}

Acevedo, O. J. F., Caballero, L. M. L. T, \& Quigora, R. J. U. (2018). Tratamiento quirúrgico del angiofibroma nasofaríngeo juvenil en pacientes pediátricos. Instituto Nacional de Saúde Infantil, 70 (5), 279-285.

Bautista, E. L., Espinoza, D. G., Rull, T. G., Pliego, E. A., \& Orozco, F. H. (2013). Angiofibroma juvenil nasofaríngeo: experiencia en un hospital general. In Anales de Otorrinolaringología Mexicana. 58(2), 79-86.

Catunda, I. V., Melo, A. R., Medeiros Júnior, R., Queiroz, I. V., Fonte Neto, A. S. \& Leão, J. C. (2011). Osteotomia le fort i: aspectos de interesse no tratamento de nasoangiofibroma juvenil. Revista de Cirurgia e Traumatologia Buco-Maxilo-Facial, 11(4), 9-12.

Chavolla-Magaña, R., Peraza-Mc-Liberty, R. A., Penagos-Noriega, S., \& Guerrero-Avendaño, G. M. (2019). Embolización preoperatoria de angiofibromas nasofaríngeos: hallazgos angiográficos y aporte vascular más frecuente. Segunda parte. Anales de Radiologia, Mexico, 18(1).

Correa, L. M. M., Renno, R. S., \& dos Reis, J. M. (2011). Nasoangiofibroma juvenil: relato de caso. Rev. Med. Minas Gerais, 21 (4), $476-478$. 
Research, Society and Development, v. 11, n. 1, e41311124800, 2022

(CC BY 4.0) | ISSN 2525-3409 | DOI: http://dx.doi.org/10.33448/rsd-v11i1.24800

Godoy, M. D. C. L., Bezerra, T. F. P., Pinna, F. R., \& Voegels, R. L. (2014). Complications in the endoscopic and endoscopic-assisted treatment of juvenile nasopharyngeal angiofibroma with intracranial extension. Brazilian Journal of otorhinolaryngology, 80 (2), 120-125.

González, A. H., López, Y. R., Sánchez, S. H., \& Ramírez, C. D. (2020). Abordaje endoscópico en el nasoangiofibroma juvenil. Jornal Cubano de Otorrinolaringologia, $21(3)$.

Hechavarría, Y. G., Torres, M. B. M., Correa, J. A. P., \& Díaz, A. P. S. (2020). Angiofibroma nasal en el adulto. Revista Cubana de Otorrinolaringología y Cirugía de Cabeza y Cuello, 4(2).

Hernández-Alfaro, F., \& Guijarro-Martínez, R. (2013). “Twist technique” for pterygomaxillary dysjunction in minimally invasive Le Fort I osteotomy. Associação Americana de Cirurgiões Bucais e Maxilofaciais.

Hintz, L. G., Penna, G. B. \& Moussalle, L. D. (2018). Fisioterapia no contexto hospitalar de um paciente pediátrico com nasoangiofibroma juvenil: relato de caso. Fundamentos e Práticas da Fisioterapia, 5(11), 95-101.

Iglesias, A. M. L., Baña, F. T. C., Hernández, M. P. R., La, M. F. M., Sánchez, R. M. V., \& Alemán, R. M. G. (2017). Angiofibroma juvenil. Gaceta Médica Espirituana, 8(2)

Kim, J. W., Chin, B. R., Park, H. S., Lee, S. H., Kwon, T. G. (2011). Cranial nerve injury after Le Fort I osteotomy. Int. J. Oral Maxillofac. Surg. 40, $327-329$.

Lin, Y. Qiu, J., Qiao, L., He, L., \& Zha, D. (2008). Le Fort I osteotomy for extensive juvenile nasopharyngeal angiofibroma: a retrospective study. Adv Ther, $25(10), 1057-1064$

Kotaniemi, K. V., Suojanen, J., \& Palotie, T. (2021). Peri-and postoperative complications in Le Fort I osteotomies. Journal of Cranio-Maxillo-Facial Surgery.

Makhasana, JAS, Kulkarni, MA, Vaze, S., \& Shroff, AS (2016). Angiofibroma nasofaríngeo juvenil. Journal of oral and maxillofacial pathology: JOMFP, 20 (2), 330 .

Martins, M. B. B., Lima, F. V. F. D., Mendonça, C. A., Jesus, E. P. F. D., Santos, A. C. G., Barreto, V. M. P., \& Santos Júnior, R. C. (2013). Nasopharyngeal angiofibroma: Our experience and literature review. International archives of otorhinolaryngology, 17, 14-19.

Mello-Filho, F. V., Araujo, F. C. F., Netto, P. B. M., Pereira-Filho, F. J. F., de Toledo-Filho, R. C., \& Faria, A. C. (2015). Resection of a juvenile nasoangiofibroma by Le Fort I osteotomy: Experience with 40 cases. Journal of Cranio-Maxillofacial Surgery, 43 (8), 1501-1504.

Peixe, C. R. (2019). Angiofibroma nasofaríngeo juvenil: perspetiva atual (Doctoral dissertation).

Quintão, L. M. V., Pereira, T. P., Leite, I. C. G., \& Urbano, E. S. (2019). Análise morfométrica maxilar aplicada a osteotomia Le Fort I. HU Hev, 45 (1), $47-$ 52 .

Quiquia, V. E. D. (2017). Nasoangiofibroma juvenil: manejo interdisciplinario de un caso. Revista Científica de Ciencias de la Salud, 10(1).

Salcedo, J. R., \& Buitrago, D. M. V. (2011). Nasoangiofibroma juvenil en un paciente adulto masculino. Acta de Otorrinolaringología \& Cirugía de Cabeza y Cuello, 39 (2), 85-90

Santos, J. A. \& Parra Filho, D. (2012). Metodologia científica. Faculdade Venda Nova do Imigrante - FAVENI.

Torres, J. G. L. (2012). Angiofibroma juvenil nasofaríngeo. Revista Cubana de Medicina General Integral, 28(2).

Viana Júnior, E. F., Cantanhede, A. L. C., Martins Neto, R. S., Moraes, R. P., Martins, B. B. \& Bastos, E. G. (2018). Osteotomia Le Fort I para tratamento cirúrgico do angiofibroma juvenil nasofaríngeo: relato de três casos. Revista de Cirurgia e Traumatologia Buco-Maxilo-Facial, 35-41. 